\title{
Three Faces of COVID-19 in Children and the Susceptibility of the Children of Healthcare Professionals
}

\author{
๑ Özgür Ceylan, ㄴ Leman Tekin Orgun, 이 Ilknur Erol
}

Department of Pediatrics, Baskent University Faculty of Medicine, Adana Dr. Turgut Noyan Teaching and Medical Research Center, Adana, Turkey

\section{ABSTRACT}

The coronavirus disease 2019 (COVID-19) is spreading around the world affecting many people and leading to serious morbidity and mortality. Detailed clinical data about COVID-19 in the adult population have been reported from different countries; however, data about COVID-19 in the pediatric population are limited with only a few reports. Three children with COVID-19 were presented to discuss different clinical and radiologic findings of COVID-19 in children in this case reports. Three consecutive children (ages 6 months, 18 months, and 3 years) were diagnosed through real-time polymerase chain reaction as being positive for severe acute respiratory syndrome coronavirus 2. Two of the patients were children of healthcare workers. Two of the cases could be classified as pneumonia; however, one of these had a severe clinical course with secondary bacterial infection, while the other was diagnosed as acute bronchiolitis. None of our patients required intensive care, mechanical ventilation, or had any severe complications. They were all discharged in good health and had no additional symptoms or signs during the follow-up period. Two of the children were the first two children from Turkey to be diagnosed COVID-19 positive, and unfortunately, two of the patients were children of healthcare workers.

Keywords: Children, COVID-19, health care professional, SARS-CoV-2

\section{INTRODUCTION}

Dr. Leman Tekin Orgun. Department of Pediatrics, Baskent University Faculty of Medicine, Adana Dr. Turgut Noyan Teaching and Medical Research Center, Adana, Turkey

Phone: +90 5056336096

E-mail:

lemantekin15@yahoo.com

Received Date: 03.09.2020

Accepted Date: 06.02.2021

Published online: 20.08 .2021

@Copyright 2021 by Anatolian Journal of Family Medicine -

Available online at

www.anatoljfm.org

OPEN ACCESS

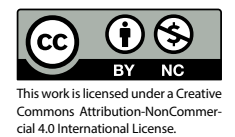

The coronavirus pandemic has affected many countries globally and leads to significant mortality and serious problems including economic and social problems. A novel coronavirus was identified in patients in an outbreak with severe pneumonia in late 2019 in Wuhan, China. ${ }^{[1,2]}$ This is a new type of coronavirus that has not been previously identified in humans. This novel coronavirus was called severe acute respiratory syndrome coronavirus 2 (SARS-CoV-2), and the official name of the disease caused by the virus was announced as coronavirus disease-19 (COVID-19) by the World Health Organization. ${ }^{[3]}$ The coronavirus is an enveloped RNA virus, and coronaviruses are a large family of viruses that cause illness ranging from the common cold to more severe diseases. ${ }^{[4,5]}$ In adults and children, coronaviruses are reported to cause respiratory, digestive, liver, and nervous system disorders. ${ }^{[5-7]}$ Although, it is known that elderly people are at higher risk for severe illness and that the pediatric population has a mild to moderate clinical manifestation, the disease can also lead to fatal consequences in children and young people with weakened immune systems. ${ }^{[8-10]}$ Detailed clinical data about COVID-19 in the adult population have been reported from different countries; however, COVID-19 data in the pediatric population are limited with only a few reports. ${ }^{[9-12]}$ On 11 March, Turkey declared its first case of coronavirus. In this article, three consecutive children with COVID-19 who had different clinical and radiologic findings are reported aiming at drawing attention to the wide clinical spectrum 
of COVID-19 in children. Besides this, two of the children we report were also the first two children in Turkey to be diagnosed as having COVID-19. In addition, two of our patients were children of healthcare professionals.

\section{CASE REPORT}

\section{Case 1}

A six-month-old male infant, who had been in contact with his COVID-positive father (a doctor), was detected as positive for SARS-CoV-2 using real-time reverse transcription-polymerase chain reaction (RT-PCR). He was quarantined with his family at home because he showed no active symptoms except mild nasal obstruction. The family, pregnancy and delivery history were unremarkable. The boy was previously healthy, and he had no history of hospitalization and underlying disease. All vaccinations of the patient had been performed as appropriate for his age. On the third day of the quarantine period, he was readmitted to the hospital with new-onset mild cough and diarrhea. The physical examination of the boy revealed a body tem- perature of $36.7^{\circ} \mathrm{C}$ with pharyngeal hyperemia, no respiratory distress, and no cyanosis. Initial workups including complete blood count, biochemistry, and acute- phase reactants were unremarkable (Table 1). Mild ground-glass opacities (GGO) on the bilateral superior zone of the lungs were noted on the initial chest X-ray image (Fig. 1a). Chest computed tomography (CT) revealed GGO in the subpleural areas of the bilateral upper and lower lobes (Fig. 1b).
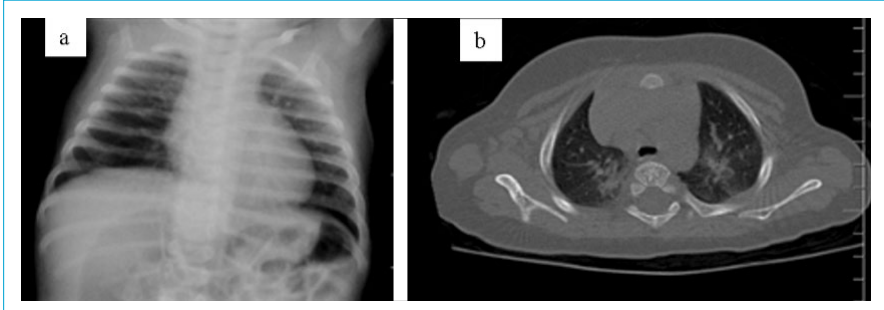

Figure 1. The chest imagings of the case 1. (a) Mild ground-glass opacities in the bilateral superior zone of the lungs in the chest X-ray. (b) Ground-glass opacities in the subpleural areas of the bilateral upper and lower lobes in the chest computed tomography.

Table 1. The demographical, clinical and laboratory value of the patients with COVID-19

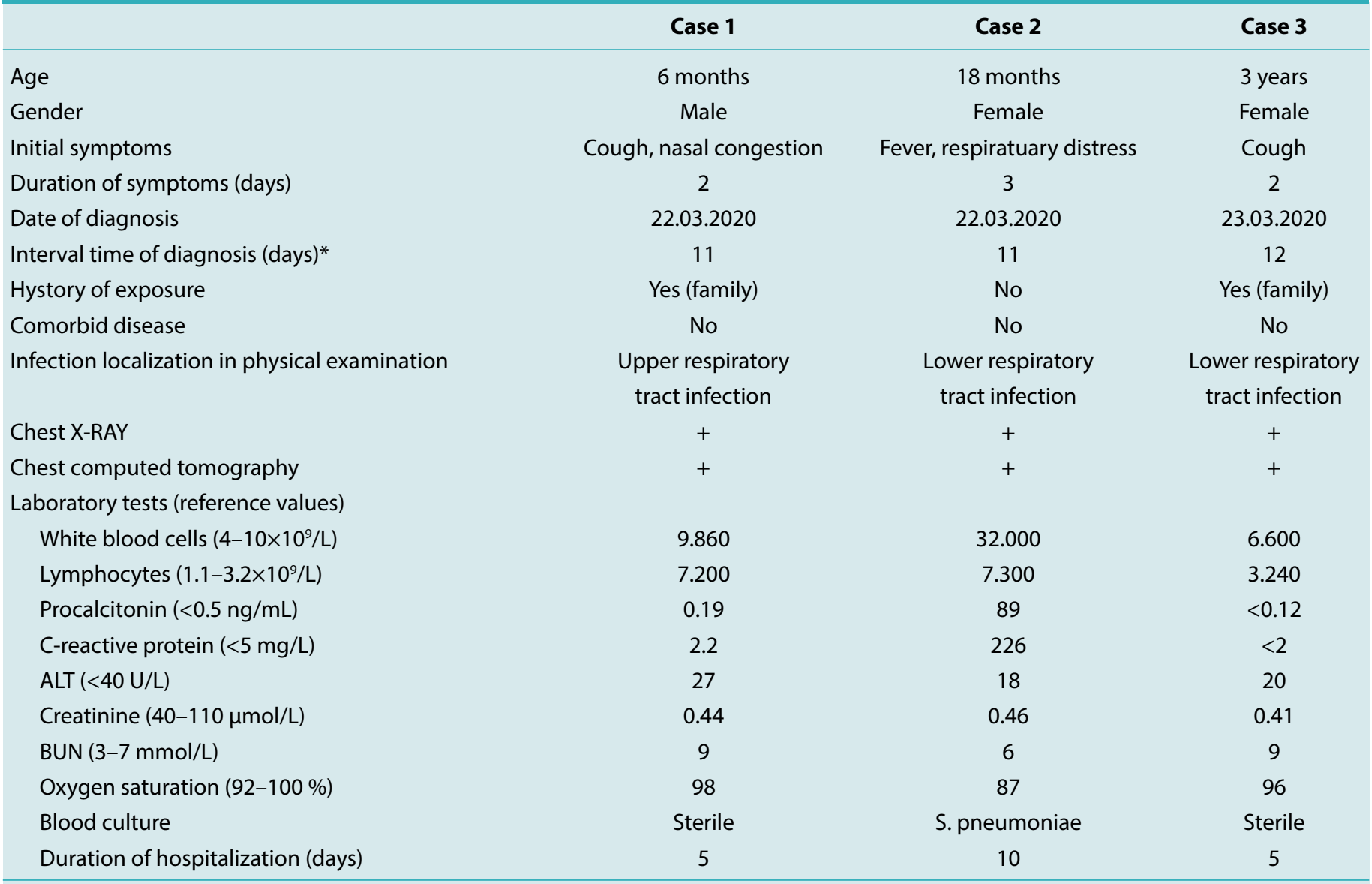

ALT: Alanine aminotransferase; BUN: Blood urea nitrogen; S. pneumoniae: Streptococcus pneumonia.

*Between the diagnosis of the patient and the time of the first cases with COVID-19 reported in Turkey. 
Consequently, the patient was diagnosed with radiologically confirmed COVID-19-related pneumonia. Cefotaxime, oseltamivir, azithromycin and chloroquine treatment was initiated after taking samples for the blood cultures and respiratory syncytial virus (RSV) and influenza samples for the respiratory virus panel. The patient had no fever, or any sign of lower respiratory tract infection and his blood culture was sterile in the follow-up of the patient. Hydroxychloroquine treatment eas discontinued according to recommendations of the Turkish Ministry of Health's COVID treatment guide in children, due to the lack of severe pneumonia in the patient. ${ }^{[13]}$ The antiviral therapy (oseltamivir) was discontinued due to the respiratory virus panel being negative for influenza. On the fifth day of hospitalization, he was discharged on oral antibiotic therapy. The condition of patient remained stable during quarantine at home.

\section{Case 2}

An 18-month-old girl was admitted to our emergency department with cough and fever for 1 week. It was learned that she received azithromycin treatment for three days. Family of the patient, pregnancy, and delivery history were unremarkable. Medical history of the patient was completely normal without any hospitalization or underlying disease. All vaccinations were also appropriate for her age. The patient was born from a twin birth and her birth weight was within the normal range. Although the patient had no history of travel and contact with a COVID-19-positive person, her older sister had otitis media 20 days ago and her twin-sister had a throat infection 1 week ago. The patient appeared languorous with an axillar temperature of $39^{\circ} \mathrm{C}$, a respiratory rate of 52 breaths per a minute, and oxygen saturation was $87 \%$ in ambient air in physical examination. Heart rate of patient was 160 beats per minute without any murmur. The patient had dyspnea and chest retraction. Chest auscultation revealed decreased breathing sounds together with bronchial breath sounds and inspiratory crepitation in the lower lobe of the left lung. The patient's hemoglobin was $9.6 \mathrm{~g} / \mathrm{dL}$, platelet count was $486.000 / \mu \mathrm{L}$, ferritin was $300 \mathrm{ng} / \mathrm{mL}$ and D-dimer was 4600 $\mu \mathrm{g} / \mathrm{L}$ (Table 1). Blood gas analysis of the patient revealed respiratory alkalosis $(\mathrm{pH}: 7.5, \mathrm{pCO} 2: 12 \mathrm{mmHg}, \mathrm{HCO}: 14.3$ $\mathrm{mEq} / \mathrm{L}$ ). The patient was hospitalized for severe pneumonia and suspicion of COVID-19. Initial chest X-ray image of the patient showed lobar consolidation and pleural effusion in the left lower lobe. Chest CT revealed patchy nodular consolidations with peripheral GGO in the subpleural areas of the right lower lobe and lobar consolidations with minimal pleural effusion of the left lower lobe (Fig. 2). The respiratory virus panel for RSV, influenza, and RT-PCR for SARS-CoV-2 from the nasopharynx were collected. Teico-

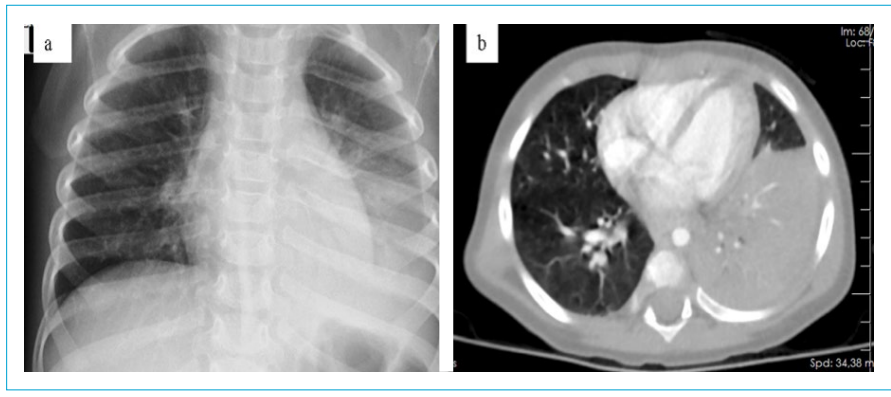

Figure 2. The chest imagings of the case 2. (a) Lobar consolidation and pleural effusion in the left lower lobe in the chest X-ray. (b) Patchy nodular consolidations with peripheral ground-glass opacities in the subpleural areas of the right lower lobe and lobar consolidations with minimal pleural effusion of the left lower lobe in the chest computed tomography.

planin, cefotaxime, oseltamivir and azithromycin treatment were initiated with oxygen support. Although the respiratory virus panel was negative for RSV and influenza, RTPCR for SARS-CoV-2 was positive. Chloroquine was added to her treatment after the positive results for SARS-CoV-2. Teicoplanin was discontinued because the blood culture that was taken on the first day of hospitalization showed penicillin-sensitive Streptococcal pneumoniae. The patient showed good clinical and radiologic improvement and became afebrile on the fourth day of treatment in the followup period. Oseltamivir was discontinued on the fifth day of therapy because the respiratory virus panel was negative for influenza. The need for oxygen support disappeared and chloroquine treatment was completed to five days following the recommendations of the current guidelines of the Turkish Ministry of Health. The patient was discharged with oral antibiotic therapy on day 10 of admission.

\section{Case 3}

A three-year-old girl was admitted to our emergency department presenting with a cough, which she had had for two days. The patient had been in contact with her mother who is a nurse. Mother of the patient was hospitalized at the city pandemic hospital with a lower respiratory tract infection due to suspected COVID-19.

Pregnancy and delivery histories were unremarkable, and birth weight was within the normal range. All vaccinations of the patient were appropriate for her age. The patient was hospitalized twice in the neonatal period with pneumonia and early infancy period with lower respiratory tract infections. Rare rales and rhonchus were heard in the left lung in the physical examination. The patient was neither dyspneic nor cyanotic with a body temperature of $37.7^{\circ} \mathrm{C}$. The patient's hemoglobin was $13.2 \mathrm{~g} / \mathrm{dL}$, platelet count was $230.000 / \mu \mathrm{L}$ and all laboratory parameters were within the normal ranges. Any infiltration was not found on the ini- 
tial chest X-ray image and chest CT was also normal (Fig. 3). The patient was hospitalized for acute bronchiolitis and suspected COVID-19, and samples for RT-PCR for SARSCoV-2 and the respiratory virus panel for RSV and influenza from the nasopharynx were collected. Her treatment was ordered as cefotaxime, oseltamivir, azithromycin and chloroquine. Although the blood culture was sterile, RT-PCR for SARS-CoV-2 was positive. The patient had no fever in the follow-up period. On the fifth day of hospitalization, respiratory findings also regressed. Chloroquine treatment was discontinued according to the recommendations of the Turkish Ministry of Health's current guidelines. Antiviral therapy was also discontinued because the respiratory virus panel was negative. On the fifth day of admission, the patient was discharged with oral antibiotic and ordered to stay in quarantine at home for 14 days.

\section{DISCUSSION}

Three children with a diagnosis of COVID-19 who had different clinical and radiologic findings are reported in this case reports, aiming to emphasize the wide clinical spectrum of COVID-19 in children, and to draw attention to the security of children of healthcare professionals. Moreover, two of the children we reported were also the first two children to be diagnosed as having COVID-19 in Turkey and unfortunately, two were children of healthcare professionals.

There have been clinical reports with large case series including detailed data of new coronavirus infections, mostly seen in adults from different countries since December 2019. ${ }^{[1-4]}$ However, data about COVID-19 in the pediatric population are limited because of the low incidence of COVID-19 in children. ${ }^{[11,13]}$ Dong et al. retrospectively evaluated the epidemiologic characteristics and transmission patterns of 2143 pediatric patients with COVID-19 in mainland China. ${ }^{[9]}$ In that study, with a median age of 7 years, $34.1 \%$ laboratory-confirmed cases and $65.9 \%$ suspected cases were reported. The authors showed that, children at

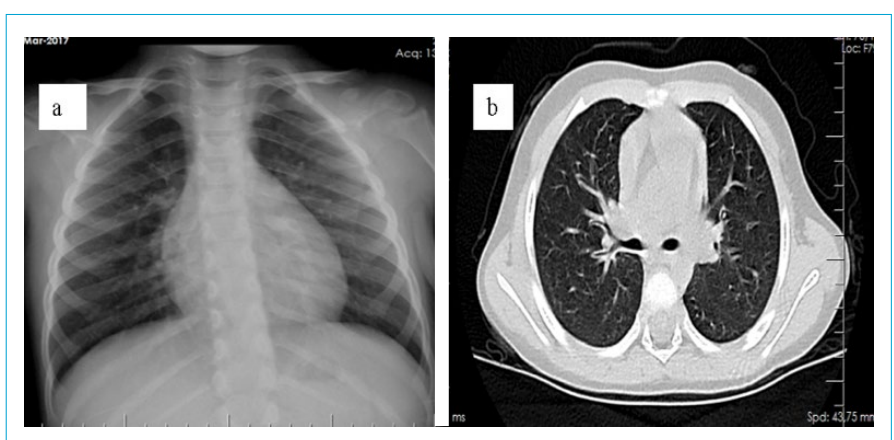

Figure 3. The chest imagings of the case 3. (a) No infiltration or abnormalities in the chest X-ray. (b) No infiltration or abnormalities in the chest computed tomography. all ages appeared to be susceptible to COVID-19, and there was no significant sex difference. ${ }^{[9]}$ However, the clinical courses of pediatric COVID-19 cases were generally less severe in children than in adults. Young children, particularly infants, were more vulnerable to COVID-19. $\cdot^{[8-10,14,15]}$ Similar$l y$, all of our patients were aged under 3 years.

Previous studies have reported the person-to-person transmission pattern of coronavirus in adults. ${ }^{[1,8,12]}$ Dong et al., also provided strong evidence on the transmission pattern of COVID-19 in children by showing exposure history with other family members and/or other children with COVID-19. ${ }^{[9]}$ This finding was also supported by Sue et al., who emphasized that all infected children had household contact with a COVID-19-positive family member. ${ }^{[14]}$ Similarly, two of our patients were diagnosed as having COVID-19 after being exposed to COVID-19-positive family members. Conversely, one of our patients had no history of contact with a person with COVID-19 and no history of travel. Moreover, all the family members had negative RT-PCR for SARS-CoV-2. Unfortunately, the family members who transmitted the virus to the first and third patients were healthcare professionals. The children of healthcare professionals might be more susceptible to diseases that cannot be prevented by vaccination during the early stages of an epidemic or pandemic. It is necessary to protect all health care providers from COVID-19. Therefore, it is very important that health authorities take a series of preventive measures such as guidance on how to use personal protective equipment properly, increase the support of logistics and providing medical equipment, and application of advanced disinfection techniques for the hotels and homes that the health care providers will reside in throughout the pandemic. Moreover, all health care providers should pay more attention to these anti-COVID-19 protective measures to prevent transmission to their family members.

The Novel Coronavirus Infection Pneumonia Diagnosis and Treatment Standards classified COVID-19 cases as mild, moderate, severe, and critical. ${ }^{[16]}$ In the literature, it was reported that children with COVID-19 had a less severe clinical course than adults. ${ }^{[2,8]}$ An observational cohort study showed that $53 \%$ of children had a moderate clinical type with pneumonia, 19\% had acute upper respiratory tract infection symptoms, classified clinically as mild disease, and $28 \%$ were asymptomatic. ${ }^{[2]}$ Similarly, Dong et al. also reported that over $90 \%$ of their children with COVID-19 were asymptomatic, mild or moderate cases. ${ }^{[9]}$ Two of our cases can be classified as having mild disease; however, according to the Novel Coronavirus Infection Pneumonia Diagnosis and Treatment Standards, the second patient should be classified as having a moderate-severe clinical course due 
to her respiratory distress with hypoxemia and resistant fever. ${ }^{[16]}$ However, none of our patients required intensive care or mechanical ventilation or had any severe complications. All the patients were discharged in good health and had no additional symptoms or signs during the follow-up period.

Although the factors that affect the severity of symptoms in individuals with COVID-19 are not known completely, it is thought that the severity of symptoms might be related to the amount of exposure, the host's own individual characteristics and underlying conditions/diseases. ${ }^{[8,9,14]}$ According to a hypothesis, the innate immune response tends to be more active in children. ${ }^{[14]}$ The other hypothesis explains the severity of the disease through angiotensin-converting enzyme II (ACE2) receptors. ${ }^{[9,14]}$ Some studies reported that SARS-CoV-2 uses the SARS-coronavirus receptor ACE2 and cellular protease TMPRSS2 (Transmembran Protease, serin 2) for entry into the cell. ${ }^{[4,17,18]}$ Therefore, it is thought that 2019-nCoV has some amino acid homology to SARS-CoV and may also be able to use ACE2 as a receptor. ${ }^{[4,17,18]}$ The causes of the severer clinical manifestations in adults with COVID-19 than that in children are also not fully understood. Although the real cause of severity in adults is not fully explained, it is known that chronic diseases such as hypertension and diabetes are more common. ${ }^{[12]}$ It is also speculated that children were less sensitive to 2019-nCoV because the maturity, function (e.g., binding ability) and number of ACE2 receptors in children may be lower than that in adults. ${ }^{[14]}$ Therefore, adults are more prone to cytokine storm, and they experience the disease more seriously. Another hypothesis is that children are usually well cared for at home and might have relatively fewer opportunities to expose themselves to pathogens and/or sick patients. ${ }^{[14]}$ Finally, because the immune system of children is still developing, it may respond to pathogens differently. ${ }^{[9,14]}$ In support of the second hypothesis, two of our patients contracted the coronavirus from their healthcare professional parents. Therefore, it is seen that being a child of a healthcare professional increases the risk of infection by COVID-19. Although it is known that the disease is milder in children aged under 10 years, our second patient had severe pneumonia findings and Streptococcus pneumonia was detected in her blood culture. Consequently, bacterial co-infection should be suspected in children with severe COVID-19 pneumonia. The most common symptoms of children with COVID-19 are acute upper respiratory tract infection symptoms including fever, fatigue, myalgia, cough, sore throat, runny nose, and sneezing. ${ }^{[8,9]}$ However, some patients may be afebrile, or have only digestive symptoms such as nausea, vomiting, abdominal pain and diarrhea. ${ }^{[8,9]}$ Early recognition of children with COVID-19 is an im- portant role to decrease disease transmission; however, it may be difficult for pediatricians and public health staff because of the mild or asymptomatic clinical process in all age groups ${ }^{[8,10]}$ According to previous reports, which studied clinical and radiological features in pediatric patients with COVID-19 infection, it is known that some children may be diagnosed only radiologically or RT-PCR for SARSCoV-2. ${ }^{[14,19]}$ The first patient was diagnosed through direct radiologic findings. Although his initial image chest $\mathrm{X}$-ray showed only suspected infiltrations on bilateral superior lobes, chest CT showed patchy consolidations-infiltrations in the subpleural areas. The diagnosis was also confirmed by RT-PCR for SARS-CoV-2.

Treatment of children with COVID-19 is achieved through symptomatic and respiratory support because there are currently no drugs available that can target SARS-CoV-2 in the pediatric population. There is currently insufficient scientific evidence for the treatment of COVID-19 infection in children. In the latest guidelines of the Turkish Ministry of Health on COVID 19 in children dated September 2020, it is stated that most of the time, supportive treatment in children is sufficient. ${ }^{[13]}$ According to the guidelines, if a child needs treatment, the treatment is planned according to the studies in adults and the severity of the child's health condition. With respect to the severity of pneumonia, although being a treatment for mild pneumonia, chloroquine (for children aged above 5 years), lopinavir/ ritonavir and/or favipiravir can be used in moderate-severe pneumonia. According to the guidelines, doses of drugs that can be used in childhood treatment and application types are given in Table 2 . All our patients received symptomatic treatment including intravenous hydration. Only one was given respiratory support with an oxygen mask. Although it is suggested to avoid the routine use of broadspectrum antibiotics in the treatment of children with COVID-19, changes in the clinical findings should be followed carefully for accompanying bacterial and fungal infections. ${ }^{[7,16]}$ In addition, samples for pathogen analysis should be collected, and timely and rational use of antibiotics should be considered as in our second case. ${ }^{[16]}$ She had severe symptoms together with coinfection of penicillin-sensitive Streptococcus pneumonia. Therefore, we believe that this coinfection led to the deterioration of the clinical course of the patient.

Three children with COVID-19 from Turkey with three different features and radiologic findings were presented and the children of healthcare professionals are at high risk during pandemic or epidemic periods was also emphasized in this case report. Therefore, it may be recommended that producing solutions for the protection of the children of 
Table 2. Doses of drugs that can be used in childhood treatment and application types

Drug Name

\section{First Choice}

Hydroxychloroquine $200 \mathrm{mg}$ tablet

In Progressive or Alternative Therapy

Lopinavir $250 \mathrm{mg} /$ ritonavir 50mg tablet

\section{Children older than 15 years}

Daily dosage and route of administration

Treatment duration (day)

1. Hydroxychloroquine sulfate; at $6.5 \mathrm{mg} / \mathrm{kg} /$ dose twice a day

5 days

(maximum dose on first day: $400 \mathrm{mg} /$ dose) on the first day

2. $3.25 \mathrm{mg} / \mathrm{kg} /$ dose twice a day per day (maximum dose

$200 \mathrm{mg} /$ dose) on the $2 \mathrm{nd}-5$ th days

1. For children between 14 days and 6 months:

10-14 days

Lopinavir component 16 mg / kg /dose twice a day

2. Between 6 months - 18 years:

15-25 kg: $200 \mathrm{mg}-50 \mathrm{mg}$ twice a day

26-35 kg: $300 \mathrm{mg}-75 \mathrm{mg}$ twice a day

>35 kg: $400 \mathrm{mg}-100 \mathrm{mg}$ twice a day

Favipiravir $200 \mathrm{mg}$ tablet

5 days

2x1600 mg loading, 2×600 mg maintenance

healthcare professionals in strict adherence with the infection control measures within the hospital, and to ensure the separate care of children from their parents. More reports containing many cases, including detailed clinical, radiological and serological data, could help improve knowledge on the characteristics of COVID-19 in the pediatric population.

\section{Disclosures}

Informed Consent: Written informed consent was obtained from the patients' family for the publication of the case report and the accompanying images.

Conflict of Interest: The authors declare that there are no conflicts of interest relevant to this study.

Peer-review: Externally peer-reviewed.

Funding: The authors received no financial support for the research, authorship, and/or publication of this article.

Authorship contributions: Concept - Ö.C., L.T.O., İ.E.; Design - Ö.C., L.T.O., İ.E.; Supervision - İ.E.; Materials - Ö.C., L.T.O.; Data collection \&/or processing - Ö.C., L.T.O.; Analysis and/or interpretation - Ö.C., L.T.O.; Literature search - Ö.C., L.T.O.; Writing - Ö.C., L.T.O.; Critical Review - I.E.

\section{REFERENCES}

1. Wu P, Hao X, Lau EHY, Wong JY, Leung KSM, Wu JT, et al. Realtime tentative assessment of the epidemiological characteristics of novel coronavirus infections in Wuhan, China, as at 22 January 2020. Euro Surveill 2020;25(3):2000044. [CrossRef]

2. Qiu H, Wu J, Hong L, Luo Y, Song Q, Chen D. Clinical and epidemiological features of 36 children with coronavirus disease
2019 (COVID-19) in Zhejiang, China: an observational cohort study. Lancet Infect Dis 2020;20(6):689-96. [CrossRef]

3. WHO. Country \& technical guidance - coronavirus disease (COVID-19). Novel Coronavirus (2019-nCoV) Situation Report 22. Available at: https://www.who.int/emergencies/diseases/ novel-coronavirus-2019/technical-guidance. Accessed Feb 11, 2020.

4. Lu R, Zhao X, Li J, Niu P, Yang B, Wu H, et al. Genomic characterisation and epidemiology of 2019 novel coronavirus: implications for virus origins and receptor binding. Lancet 2020;395:565-74. [CrossRef]

5. Su S, Wong G, Shi W, Liu J, Lai ACK, Zhou J, et al. Epidemiology, genetic recombination, and pathogenesis of coronaviruses. Trends Microbiol 2016;24(6):490-502. [CrossRef]

6. Wang D, Hu B, Hu C, Zhu F, Liu X, Zhang J, et al. Clinical characteristics of 138 hospitalized patients with 2019 novel coronavirus-infected pneumonia in Wuhan, China. JAMA 2020;323(11):1061-9. [CrossRef]

7. Heimdal I, Moe N, Krokstad S, Christensen A, Skanke LH, Nor$\mathrm{db} \varnothing \mathrm{SA}$, et al. Human coronavirus in hospitalized children with respiratory tract infections: a 9-year population-based study from Norway. J Infect Dis 2019;219(8):1198-206. [CrossRef]

8. CDC COVID-19 Response Team. Coronavirus disease 2019 in children - United States, February 12-April 2, 2020. MMWR Morb Mortal Wkly Rep 2020;69(14):422-6. [CrossRef]

9. Dong Y, Mo X, Hu Y, Qi X, Jiang F, Jiang Z, et al. Epidemiology of COVID-19 among children in China. Pediatrics 2020;145(6):e20200702. [CrossRef]

10. Lu X, Zhang L, Du H, Zhang J, Li YY, Qu J, et al; Chinese Pediatric Novel Coronavirus Study Team. SARS-CoV-2 Infection in Children. N Engl J Med 2020;382(17):1663-5. [CrossRef] 
11. Park JY, Han MS, Park KU, Kim JY, Choi EH. First pediatric case of coronavirus disease 2019 in Korea. J Korean Med Sci 2020;35(11):e124. [CrossRef]

12. Guan WJ, Ni ZY, Hu Y, Liang WH, Ou CQ, He JX, et al. Clinical characteristics of Coronavirus disease 2019 in China. N Engl J Med 2020;382(18):1708-20. [CrossRef]

13. T.C. Sağlık Bakanlığı Halk Sağlığı Genel Müdürlüğü. COVID-19 (SARS-CoV-2 Enfeksiyonu) çocuk hasta yönetimi ve tedavi. Bilimsel danışma kurulu çalışması. Ankara: T.C. Sağlık Bakanlığı; 2020.

14. Su L, Ma X, Yu H, Zhang Z, Bian P, Han Y, et al. The different clinical characteristics of corona virus disease cases between children and their families in China - the character of children with COVID-19. Emerg Microbes Infect 2020;9(1):707-13. [CrossRef]

15. Cruz AT, Zeichner SL. COVID-19 in Children: initial characteriza- tion of the pediatric disease. Pediatrics 2020;145(6):e20200834. 16. Diagnosis and Treatment Protocol for Novel Coronavirus Pneumonia (Trial Version 7). Chin Med J (Engl) 2020;133(9):108795. [CrossRef]

17. Li W, Moore MJ, Vasilieva N, Sui J, Wong SK, Berne MA, et al. Angiotensin-converting enzyme 2 is a functional receptor for the SARS coronavirus. Nature 2003;426(6965):450-4. [CrossRef]

18. Hoffmann M, Kleine-Weber H, Schroeder S, Krüger N, Herrler T, Erichsen S, et al. SARS-CoV-2 cell entry depends on ACE2 and TMPRSS2 and is blocked by a clinically proven protease inhibitor. Cell 2020;181(2):271-80.e8. [CrossRef]

19. Xia W, Shao J, Guo Y, Peng X, Li Z, Hu D. Clinical and CT features in pediatric patients with COVID-19 infection: Different points from adults. Pediatr Pulmonol 2020;55(5):1169-74. [CrossRef] 